\title{
The Peculiar Logic of the Black-Scholes Model
}

\author{
James Owen Weatherall \\ Department of Logic and Philosophy of Science \\ University of California, Irvine
}

\begin{abstract}
The Black-Scholes(-Merton) model of options pricing establishes a theoretical relationship between the "fair" price of an option and other parameters characterizing the option and prevailing market conditions. Here I discuss a common application of the model with the following striking feature: the (expected) output of analysis apparently contradicts one of the core assumptions of the model on which the analysis is based. I will present several attitudes one might take towards this situation, and argue that it reveals ways in which a "broken" model can nonetheless provide useful (and tradeable) information.
\end{abstract}

Keywords: Black-Scholes model, Black-Scholes formula, Volatility smile, Economic models, Scientific models

\section{Introduction}

The Black-Scholes(-Merton) (BSM) model (Black and Scholes, 1973; Merton, 1973) establishes a relationship between several parameters characterizing a class of financial products known as options on some underlying asset. ${ }^{1}$ From this model, one can derive a formula, known as the Black-Scholes formula, relating the theoretically "fair" price of an option to other parameters characterizing the option and prevailing market conditions. The model was first published by Fischer Black and Myron Scholes in 1973, the same year that the first U.S. options exchange opened, and within a decade it had become a central part of

\footnotetext{
Email address: weatherj@uci.edu (James Owen Weatherall)

${ }^{1}$ I will return to the details of the BSM model below, including a discussion of what an "option" is. The same model was also developed, around the same time and independently, by Merton (1973); other options models with qualitatively similar features, resulting in essentially the same pricing formula, were developed earlier, by Bachelier (1900) and Thorp and Kassouf (1967), but were much less influential, in part because the arguments on which they were based made too little contact with mainstream financial economics. For a history of these ideas, including a discussion of the origins of the BSM model, see Weatherall (2013) and references therein; see MacKenzie (2006) for a detailed discussion of the reception and impact of the model.
} 
financial practice, not only as the theoretical basis for essentially all options trading, but as the theoretical gold-standard on which many other asset pricing models were based. The BSM model and its close descendants are widely used by financial practitioners today.

The BSM model, like any model in any discipline, is based on a number of assumptions. ${ }^{2}$ Also like many models in many disciplines, it is regularly used despite the fact that these assumptions are often controversial, or known to hold at best approximately. ${ }^{3}$ But the BSM model is (arguably) unusual in at least one striking way: in a principal application, the output of the model explicitly and reliably contradicts one of its core assumptions.

One might expect this sort of contradiction to undermine the success or reliability of the model, or at least to speak against its use. But that is not the reaction in practice. In what follows, my goal will be to draw out this peculiar situation, and to discuss some attitudes that one might take to it. I will ultimately argue that the continued use of the Black-Scholes model, in the particular application I will describe, is both justified and fruitful, despite its strange features. Ultimately the model is useful because it provides a way of measuring the degree to which its own assumptions fail, which in turn provides new insight into market conditions. I take this to reveal an important wrinkle in debates concerning the "realism" of assumptions in economic models: at least in this case, there is a subtle and intricate interplay between an unrealistic assumption and the application of a model, which allows practitioners to use the model successfully despite its lack of verisimilitude.

The remainder of this paper will be structured as follows. I will first present some background on options and the BSM model, including a discussion of the assumptions in

\footnotetext{
${ }^{2} \mathrm{My}$ purpose is to draw out and analyze the unusual features of this particular model (in application); I will not engage in detail with the large literature on scientific models in general, or even economic models in particular. For further details on these subjects, one should consult Weisberg (2013), Boland (2014), and references therein. As a rule, I tend to be skeptical of the idea that scientific models in general (as opposed to collections of saliently similar models) are a fruitful unit of analysis; for a defense, see O'Connor and Weatherall (2016).

${ }^{3}$ Fischer Black himself published a critical discussion of the assumptions underlying his eponymous model, under the title "The Holes in Black-Scholes" (Black, 1988).
} 
the model. In the next section, I will describe some applications of the model, and draw out the sense in which, in one of its principal applications, the BSM model appears to contradict itself. I will then present three attitudes one might take to this situation. I will conclude with some brief remarks about how this example bears on issues in philosophy of economic modeling.

\section{The Black-Scholes-Merton Model}

A (European, call) option is a contract that entitles (but does not obligate) the holder to buy an asset (the underlying asset) at a pre-determined price (the strike price) at some fixed time in the future (expiration). ${ }^{4}$ One may think of an option as a bet that the market price for the underlying will exceed the strike price at expiration, with the payoff determined by the difference between that market price and the strike price. This is because if the market price exceeds the strike price at expiration, one can exercise the option and then sell the asset on the open market, pocketing the difference.

The problem of options pricing is to determine how much one should be willing to pay now for an option with a given strike price and expiration, on an underlying asset with given current market price. The BSM model provides a solution to the problem of options pricing. ${ }^{5}$

The BSM model is based on four main assumptions. ${ }^{6}$

\footnotetext{
${ }^{4}$ Variations on this are possible. For instance, a put option (as opposed to a call option) is a contract entitling the holder to sell an underlying asset. An American option (as opposed to a European option) allows the holder to exercise the option at any time before expiration, as opposed to at expiration. All of these are so-called "vanilla" options; "exotic" options, which include various further complications, are also traded.

${ }^{5}$ The treatment of the BSM model here follows Derman and Miller (2016); see also Cox and Rubinstein (1985) and Chriss (1997) for further discussion of the BSM model and related derivatives pricing models.

${ }^{6}$ There are other assumptions, too: no bid-ask spreads, no forced unwindings, etc. A detailed discussion of the assumptions behind the model is given by Black (1988); see also Black (1989).
} 
1. (GBM) The underlying asset price $S$ satisfies the stochastic differential equation

$$
d S=\mu S d t+\sigma S d Z
$$

Here $Z$ is a Wiener process, $\mu$ is the (constant) percentage drift, and $\sigma$ is the (constant) percentage volatility.

2. (Ito) The price $C(S, t)$ of an option is a twice differentiable function of $S$ and $t$.

3. (DH) Traders can trade continuously and take arbitrarily large long and short positions with no transaction costs.

4. (CAPM) There exist risk-free assets, and all such assets earn a fixed (known) rate of return $r$.

From these assumptions, one can derive a formula relating the price $C$ of an option to the other parameters just described.

The derivation proceeds as follows. Invoking (GBM) and (Ito), one can apply a standard result in the theory of stochastic differential equations, known as Ito's lemma, to derive a stochastic differential equation for $C:^{7}$

$$
d C=\left(\frac{\partial C}{\partial t}+\mu S \frac{\partial C}{\partial t}+\frac{1}{2}(\sigma S)^{2} \frac{\partial^{2} C}{\partial S^{2}}\right) d t+\frac{\partial C}{\partial t} \sigma S d Z
$$

The stochastic term $Z$ in Eq. (1) is the same Wiener process that drives the evolution of $S$; this means that one can construct, at any time, an instantaneous portfolio, consisting of positions in the option and its underlying asset, such that the $d Z$ terms cancel. But an asset - or, in this case, a portfolio - whose price has no stochastic contribution is, by definition, risk-free: there is no uncertainty in its evolution. Hence, by invoking (CAPM), we may infer that this risk-free portfolio must earn the risk-free rate of return $r$.

\footnotetext{
${ }^{7}$ For background on stochastic calculus, see, for instance, Oksendal (2010).
} 
These arguments lead us to the Black-Scholes equation:

$$
\frac{\partial C}{\partial t}+r S \frac{\partial C}{\partial S}+\frac{1}{2} \sigma^{2} S^{2} \frac{\partial^{2} C}{\partial S^{2}}=r C
$$

Crucially, the Black-Scholes equation is a partial differential equation, not a stochastic differential equation; thus it admits solutions that are ordinary functions of real variables.

The Black-Scholes formula is one such solution to the Black-Scholes equation. It may be written as:

$$
C(S, K, \tau, \sigma, r)=\frac{1}{2 \pi}\left(S \int_{-\infty}^{d_{1}} e^{-\frac{1}{2} y^{2}} d y-K e^{-r \tau} \int_{-\infty}^{d_{2}} e^{-\frac{1}{2} y^{2}} d y\right)
$$

where

$$
\begin{aligned}
& d_{1}=\frac{\ln \frac{S}{K e^{-r \tau}}+\frac{1}{2} \sigma^{2} \tau}{\sigma \sqrt{\tau}} \\
& d_{2}=\frac{\ln \frac{S}{K e^{-r \tau}}-\frac{1}{2} \sigma^{2} \tau}{\sigma \sqrt{\tau}} .
\end{aligned}
$$

Here $K$ is the strike price of the option and $\tau$ is the time to expiration; these arise as constants of integration, fixed by the boundary conditions of the problem.

\section{The Volatility Smile}

When the BSM formula was first published in 1973, options trading was a small, unsystematic business. The first U.S. options exchange opened, in Chicago, only that year; even then, the number of assets on which options were traded in the exchange was small. Before the exchange opened, options trading was done "over the counter", i.e., in individual transactions, with little transparency or price consistency. There was no widely accepted method for pricing options, and little understanding of their value.

In this context, the BSM formula had a natural application: it could be used to calculate 
the "ideal" options price $C$, using options parameters $K$ and $\tau$, market values $S$ and $r$, and one's expectations about (or historical values of) $\sigma$. This calculation could then provide a baseline against which market prices might be compared, so that one could identify options that were over- or under-priced. This strategy was profitable until the early 1980s, by which time market prices aligned with BSM prices (MacKenzie, 2006), meaning that comparatively little advantage could be gained merely by using the BSM formula. (Still, the BSM formula, and closely related ones, continued to be used to price options - particularly by traders looking to use options to hedge their positions.)

Around this time, a second application of the BSM formula emerged. The idea behind this application was to effectively invert the formula: use market values for $C, S$, and $r$ to compute $\sigma$. The idea is that some of the parameters going into the BSM formula are readily observable: they are either parameters governing the option under consideration, such as $K$ and $\tau$, or else they are market prices, such as $C, S$, and $r$. But the future volatility of the underlying asset is not readily observable; at most, one can form an expectation of future volatility based on past volatility. Inverting the BSM formula allows one to compute, based on various observable quantities, a value for the unobservable quantity of future volatility. The value computed in this way is known as implied volatility.

Implied volatility turns out to be a useful notion. On the one hand, it provides some insight into market expectations concerning the future volatility of an asset. Perhaps more usefully still, implied volatility relativizes the price of an option to other (evolving) quantities, including the price of the underlying asset and the strike price of the option. For this reason, it provides a useful substitute for price, encoding information about how expensive an option is relative to other quantities of interest. (Briefly, the higher the implied volatility, the more expensive the option.) Options traders regularly quote implied volatilities to one another when describing trades.

But as useful as implied volatility is, it also leads to problems. In particular, the BSM 
model makes strong assumptions about the value of $\sigma$. In particular, recall that assumption (GBM) says that $S$ satisfies a stochastic differential equation:

$$
d S=\mu S d t+\sigma S d Z
$$

where $\mu$ and $\sigma$ are constant. Thus, $\sigma$ is a parameter that characterizes the underlying asset and not a particular option. It follows that (GBM) requires $\sigma$ to be independent of options parameters $K$ and $\tau$, since the options parameters concern only the details of a given option written on an asset, whereas $\sigma$ describes the dynamics of the asset itself.

During the 1980s, when implied volatility was first used, it was (essentially) independent of $K$ and $\tau$, as expected. But following the October 19, 1987 market crash - a crash in which the BSM model was broadly implicated - options markets began to change (MacKenzie, 2006; Derman and Miller, 2016). In short, traders began to notice a surprising relationship between the implied volatility of an option and the parameters characterizing that option. For fixed time to expiration, options with strike prices far from the current price-i.e., options that would only be exercised if prices changed dramatically - were observed to have higher implied volatilities. ${ }^{8}$ In other words, the more unlikely it was for an option to become valuable, the more expensive it became (as measured by the implied volatility). This anomalous behavior became known as the volatility smile.

The volatility smile was recognized as very strange. On its face, it suggests a contradiction in the BSM model - or at least, between the BSM model and the results of this application. $^{9}$ The BSM model begins by assuming that a certain parameter has a constant value. From this assumption, one can derive a relationship - the BSM formula-between that parameter and some other market parameters. But on observing market conditions,

\footnotetext{
${ }^{8}$ The generic "smile" is a parabola with minimum value at the current market price, but lots of variations are possible and the smile often looks more like a "smirk". There are also unexpected dependencies on other parameters, such as time to expiration and whether the option is a put or call.

${ }^{9}$ If you are worried that this is not really a contradiction, I am sympathetic - see the next section.
} 
one discovers - using the BSM formula - that the assumption underlying the formula does not hold, apparently undermining the formula with which the parameter was calculated. At very least, the result of this analysis reveals that the relationship captured by the BSM formula does not accurately capture the relationship between future volatility, price of the underlying asset, and the price of an option in real markets.

Nonetheless, traders continue to use the BSM model, or close descendants, for many purposes - including to calculate implied volatilities! The issue is not that the tension between the model's assumptions and its output has gone unrecognized - to the contrary, it is well-known. In a recent book, Emanuel Derman - a former physicist who, during the 1980s and 1990s, became an influential market modeler while working for Goldman Sachs (where he was a colleague of Fischer Black), and who eventually won the Financial Engineer of the Year award for his work on the volatility smile - and Michael Miller, an options trader, sums it up as follows.

The existence of the smile poses a deep and interesting problem for option valuation, in both theory and practice. In the BSM model, the volatility is the constant future volatility of a stock assumed to be undergoing geometric Brownian motion. In the BSM model, therefore, a stock must have a definite volatility. If the model accurately describes stocks and the options written on them, all options should "see" the same volatility of the underlying stock. Thus, the implied volatilities deduced from market prices of all options with the same underlier and expiration date should be the same, and the volatility smile should be perfectly flat. The nonflat smiles [seen in real markets] tells us that actual option markets are more complicated than we've been assuming, and that their prices violate the BSM model. Nevertheless, traders everywhere use implied BSM volatilities to quote options prices. The widespread use of BSM implied volatilities on trading desks, even in the presence of a BSM-violating smile, is evidence of the model's enormous persuasive and practical success. It's strange and mysterious that markets use a model that doesn't work to quote prices that deviate from it. (Derman and Miller, 2016, p. 133)

Strange and mysterious indeed. 


\section{Why Use a Broken Model?}

The upshot of the previous section — nicely summarized by Derman and Miller - is that there is a sense in which the BSM model is broken. And yet, the model continues to be used, despite this anomalous behavior. Worse, it is used for precisely the purposes that reveal its problems. What can account for this "strange and mysterious" practice?

In what follows, I will present and discuss three attitudes that one might take to the volatility smile. I take it that each of these attitudes occurs among traders and quantitative analysts - though I do not have any evidence for this aside from anecdotes from my own experience. (I do not claim that there are no other possible attitudes.)

The first attitude is the view that the BSM model is not broken after all; rather, the volatility smile is compatible with (GBM), properly construed. I will call this attitude compatibilism. The idea is that one needs to draw an important distinction between two quantities. One of these quantities is the implied volatility, which measures what market participants expect about future volatility; the other is actual volatility, which concerns the degree of variability that will in fact be exhibited by future returns. ${ }^{10}$

It follows, on this attitude, that the volatility smile is not a strict contradiction. It is actual volatility that figures into (GBM), whereas only implied volatility is found to depend on the options parameters. It follows that (GBM) is unaffected by the volatility smile. Indeed, one could accept all of the assumptions of the BSM model, but take the volatility smile to show that market participants trading options with different parameters tend to have diverse beliefs about future volatility - for instance, people interested in trading options that are unlikely to be valuable may have unusually high credences about large market moves. It is also possible that various facts about supply and demand for options with various parameters have distorted market values, say because some traders are using options to

\footnotetext{
${ }^{10}$ One can think of this as an epistemology/metaphysics distinction, insofar as implied volatility reflects market beliefs, while actual volatility reflects future facts.
} 
hedge large positions, and are willing to pay a premium to do so.

Compatibilism is probably tenable - and, the core insight, that there is not a strict contradiction, is surely correct. But the further view that, because of this core insight, the BSM model is not "broken" has some unattractive features that make it an implausible explanation of why the model continues to be used. For instance, one way to make sense of the position is to adopt a view of probability according to which there is a fact about the distribution of the random variable from which future market returns will be drawn. ${ }^{11}$ Moreover, (GBM) implies that this must be a normal distribution with some given timedependent variance, $\sigma^{2} t$, where $\sigma$ is the parameter that should appear in the BSM formula. The compatibilist would then say that although there is some fact about what this parameter is, market participants do not know it, or at least, do not trade in a way consistent with any single value for it. But this very broad disagreement might itself be taken as evidence that it is difficult to determine the correct value of $\sigma$. In other words, on this version of compatibilism, the BSM model is not incoherent, but it also is not useful, because it requires one to know something that is (apparently) very difficult to accurately determine, and perhaps in principle unknowable.

This broad disagreement about $\sigma$ reflected in the volatility smile can be interpreted in another way: one might think that the volatility smile is incompatible with a single value of $\sigma$ because market participants generally do not believe there is any value of $\sigma$ that correctly reflects future returns. In other words, market participants generally reject (GBM), and the BSM model along with it. Of course, an individual compatibilist can accept this, and even continue to use the BSM model because she happens to disagree with other market participants. But this cannot account for the widespread use of the model, since after all, the whole point would be that most traders - including many who use the BSM model - believe

\footnotetext{
${ }^{11}$ There is a more epistemic version of this view, where one says that one says that one's beliefs about future returns are well-summarized by a time-dependent normal distribution with variance $\sigma^{2} t$. This version is probably on sounder conceptual footing, but the issues discussed above are unchanged.
} 
the BSM model to be broken.

But if this is right, why is the BSM model still widely used? One answer reflects a second possible attitude towards the model, which I will call the trading zone (TZ) attitude. $^{12}$ According to this attitude, one might accept that the BSM model makes incorrect assumptions about the probabilities of future returns, and therefore should not be taken to provide reliable guidance on the relationship between options prices and other market parameters. Nonetheless, one might think that the model's conceptual framework is still useful for reasoning about and discussing options.

One reason to think it remains useful is that it draws attention to market parameters that turn out to be relevant to options pricing, even if the particular relationship between them captured by the BSM formula is not correct. These include obvious parameters - such as the price of the underlying asset-but also less obvious parameters, such as the various partial derivatives appearing in Eq. (1). Indeed, these partial derivatives - known as the Greeks, for the letters used to denote them-are widely used by traders and analysts for evaluating possible trades.

Picking out salient parameters is particularly valuable in this context, because even though there seems to be broad agreement that the BSM model is incorrect, there is no universally accepted alternative. This means that many traders use different models from one another; these traders may nonetheless communicate with one another using the language of the BSM model. More, many firms use proprietary derivatives models, and take the details of their models to have value that would be compromised if the models were revealed. But options traders and analysts often need to communicate with traders at other firms in order to execute trades. The BSM model, including the Greeks, provides a firm-independent baseline that traders and brokers can use to communicate, without revealing any proprietary

\footnotetext{
${ }^{12}$ The expression "trading zone" is a reference to Galison (1997), who coined the term to describe the ways in which scientists from different cultures - such as theoretical and experimental particle physics - manage to communicate with one another. But I do not mean to invoke any details of Galison's account.
} 
trading information. This provides a strong reason to continue to use the BSM modelparticularly for purposes such as quoting implied volatility as a substitute for price - even though everyone agrees that it fails in important cases. It provides resources for fine-grained reasoning about options, without revealing private information about the details of your own options pricing scheme.

The TZ attitude provides one answer for why the BSM model continues to be widely used, even if most market participants reject it. But there is a second, different answer to the same question that is in many ways more interesting. On this view-which I will call the informativist attitude - although one accepts that the BSM model is broken, one nonetheless takes the volatility smile to provide useful information about future probabilities. By studying, for a particular option, the way in which the BSM model fails - as reflected by the volatility curves associated with that option - one can extract useful, often tradeable, information about markets. On this view, the BSM model again acts as a kind of baseline, not for communicating across different firms, but relative to which actual market conditions can be evaluated.

To see how this might work, consider that generic volatility smiles reveal that options that are less likely to be profitable are comparatively more expensive. This alone suggests not only that the assumptions underlying the BSM model are rejected, but also why they are rejected. Specifically (GBM) implies that returns will be normally distributed with variance $\sigma^{2} t$. It is this assumption that is rejected, because market participants tend to believe that large changes in the underlying price are more likely than (GBM) predicts. In other words, the volatility smile may be taken as an indication that market participants believe the distribution over future returns has excess kertosis-i.e., more weight in its tails than a normal distribution. ${ }^{13}$

\footnotetext{
${ }^{13}$ As it happens, this belief is well-supported by market data: see, for instance, Cont (2001). It has also been defended for a long time (Mandelbrot, 1963; Fama, 1963, 1965), though it did not gain wide acceptance by mainstream market participants until the late 1980s, around the same time that the volatility smile was
} 
In fact, if one interprets the volatility smile in this way, then the details of the volatility smile for a particular asset provide new information about the option and its underlying asset - or at least, new information about market expectations. This is because one can infer from the volatility smile an implied (non-normal) distribution governing the probabilities of (percentage) price changes for the underlying asset (Derman and Miller, 2016). This is detailed information that probes market expectations about the probabilities for specific ranges of returns.

\section{Conclusion}

I have now presented the BSM model and the unexpected pattern (i.e., the volatility smile) that one finds when one uses the BSM equation to calculate implied volatility. I have described why the volatility smile appears to contradict the very model used to calculate it, and I have presented three attitudes that one might take towards the volatility smile that could explain why one would nonetheless continue to use the BSM model. These attitudes are not necessarily incompatible - in particular, one could certainly accept both the trading zone attitude and the informativist attitude - but they do track importantly different considerations, any of which could explain individual traders continuing to use the BSM model despite the volatility smile.

The first attitude - compatibilism - reflects a quotidian picture of why one might use a model: because one thinks its assumptions accurately (or approximately) capture the situation one is interested in. But the situation with the other two attitudes is different. By adopting either of TZ or informativism one continues to use a model that one believes to fail, in precisely the domain where it does fail. On TZ, one does so because the BSM model can still be helpful in facilitating reasoning and communicating about options - even without

first recognized. See Bandelj et al. (2016) for a discussion of some of the conceptual issues surrounding heavy tailed distributions over returns. 
accurately capturing the relationships of interest. This attitude seems to best explain the use of implied volatilities as a substitute for price: implied volatility effectively encodes price relative to other parameters, in a way that does not require one to divulge private information. On the informativist attitude, meanwhile, one actively exploits the model's failures to extract new information about other market participants. In this way, the BSM model, by virtue of how it fails, provides a new kind of tool for understanding markets.

This third attitude reveals some important lessons for philosophy of economics - and philosophy of science more generally. The first lesson concerns the role of realistic assumptions in economics modeling. There is a long debate, going back to Friedman (1953) and others, concerning whether the standard assumptions in economic models - rational agents, efficient markets, and so on-are sufficiently realistic to support reliable inferences about, for instance, policy interventions. The example of the BSM model, at least on the informativist attitude, puts a wrinkle in this story. In the BSM model, one adopts an assumptions that is widespread - and regularly criticized as unrealistic. (Indeed, (GBM) may even be understood as a consequence of the efficient markets hypothesis.) But one does not actually expect this assumption to hold. Instead, one uses the model to gauge the degree to which the assumption fails and to measure the details of how it fails. ${ }^{14}$ This suggests that bad assumptions do not always lead to useless models.

A second lesson is related to an idea often defended by Bob Batterman, which is that the circumstances under which a model breaks down are often a valuable output. For instance, he writes:

...we [should] rethink the use of models in physics. If we include mathematical features as essential parts of physical modeling then we will see that blowups or singularities are often sources of information. (Batterman, 2011, p. 1038)

\footnotetext{
${ }^{14}$ There is an interesting connection here to recent work by Jenn Jhun (2017), who has argued that the "unrealistic" assumptions of equilibrium economics set a baseline of ideal behavior that allows one to better reason about realistic (i.e., non-ideal) behavior.
} 
The present example is different in many details from the examples he considers - which generally involve singular behavior near critical points and applications of renormalization group theory - but I think it is similar in spirit: it is because the BSM model fails that it turns out to be so useful. ${ }^{15}$

\section{Acknowledgments}

This paper is partially based upon work supported by the National Science Foundation under

Grant No. 1328172. I am grateful to the audience at PSA 2016 and to my co-symposiasts for helpful comments and discussion; and to an audience at Cambridge HPS for their comments on a related talk.

\section{References}

Bachelier, L., 1900. Théorie de la spéculation. Gauthier-Villars, Paris.

Bandelj, N., Elyachar, J., Richardson, G., Weatherall, J. O., 2016. Comprehending and regulating financial crises: An interdisciplinary approach. Perspectives on Science 24 (4), 443-473.

Batterman, R. W., 2011. Emergence, singularities, and symmetry breaking. Foundations of Physics 41 , 1031-1050.

Black, F., 1988. The holes in Black-Scholes. Risk 1 (4), 30-33.

Black, F., 1989. How to use the holes in Black-Scholes. Journal of Applied Corporate Finance 1 (4), 67-73.

Black, F., Scholes, M., 1973. The pricing of options and corporate liabilities. Journal of political economy 81 (3), 637-654.

Boland, L. A., 2014. Model Building in Economics: its purposes and limitations. Cambridge University Press, Cambridge, UK.

Chriss, N. A., 1997. Black-Scholes and Beyond: Option Pricing Models. McGraw-Hill, New York, NY.

Cont, R., 2001. Empirical properties of asset returns: stylized facts and statistical issues. Quantitative Finance 1, 223-236.

Cox, J. C., Rubinstein, M., 1985. Options Markets. Prentice-Hall, Englewood Cliffs, NJ.

Derman, E., Miller, M. B., 2016. The Volatility Smile. Wiley, Hoboken, NJ.

Fama, E. F., 1963. Mandelbrot and the stable Paretian hypothesis. The Journal of Business 36 (4), $420-429$.

Fama, E. F., 1965. The behavior of stock-market prices. The Journal of Business 38 (1), 34-105.

Friedman, M., 1953. Essays in Positive Economics. University of Chicago Press, Chicago, IL, Ch. The Methodology of Positive Economics, pp. 3-43.

Galison, P., 1997. Image and logic: A material culture of microphysics. University of Chicago Press, Chicago, IL.

Jhun, J., 2017. What's the point of ceteris paribus? (or: How to understand supply and demand curves), forthcoming in Philosophy of Science.

\footnotetext{
${ }^{15}$ That said, there are other examples of financial models in which break-downs and blow-ups are sources of information that are much closer to the examples Batterman discusses: see, for instance, Johansen et al. (2000) and Jhun et al. (2017).
} 
Jhun, J., Palacios, P., Weatherall, J. O., 2017. Critical market crashes? explanation, idealization, and universality in econophysics, forthcoming in Synthese. arXiv:1704.02392 [physics.hist-ph].

Johansen, A., Ledoit, O., Sornette, D., 2000. Crashes as critical points. International Journal of Theoretical and Applied Finance 3 (2), 219255.

MacKenzie, D., 2006. An Engine, Not a Camera. MIT Press, Cambridge, MA.

Mandelbrot, B., 1963. The variation of certain speculative prices. The Journal of Business 36 (4), 394-419.

Merton, R. C., 1973. Theory of rational option pricing. The Bell Journal of economics and management science, 141-183.

O'Connor, C., Weatherall, J. O., 2016. Black holes, black scholes, and prairie voles: An essay review of simulation and similarity, by michael weisberg. Philosophy of Science 83 (4), 613-626.

Oksendal, B., 2010. Stochastic Differential Equations: An Introduction with Applications, 6th Edition. Springer, Dordrecht.

Thorp, E. O., Kassouf, S. T., 1967. Beat the market: a scientific stock market system. Random House, New York, NY.

Weatherall, J. O., 2013. The Physics of Wall Street. Houghton Mifflin Harcout, Boston, MA.

Weisberg, M., 2013. Simulation and Similarity. Oxford University Press, Oxford, UK. 